Revista de Investigación Universitaria, 2015, Vol. 4 (2): 50-56

ISSN: 2312-4253 (Versión impresa) ISSN: 2078-4015 (Versión digital)

\title{
Estrés y consumo de alcohol en trabajadoras sexuales
}

\section{Stress and consumption of alcohol in sex workers}

\author{
Mamani Benito, Oscar Javier; Coaquira Pinto, Jessica Paola; Sapillado Condori, Martha \\ Escuela Profesional de Psicología, Facultad de Ciencias de la Salud, Universidad Peruana Unión \\ Recibido el 10 de mayo del 2016-Aceptado el 26 de junio del 2016
}

\begin{abstract}
Resumen
El objetivo de esta investigación fue determinar la relación entre estrés y consumo de alcohol. La metodología corresponde a un diseño no experimental de tipo descriptivo correlacional, en el cual se implica a una población conformada por 150 trabajadoras sexuales que acuden al Centro de Referencia de Infecciones de Transmisión Sexual CERITS del Hospital Carlos Monge Medrano de la ciudad de Juliaca; sin embargo, se optó por trabajar con una muestra de 103 participantes elegidas mediante un muestreo no probabilístico. Los instrumentos utilizados fueron el cuestionario de estrés y el cuestionario de identificación de trastornos debido al consumo de alcohol AUDIT, ambos validados para los objetivos de esta investigación. Los principales resultados demuestran que el $43.7 \%$ presenta estrés muy alto y el 39.8\% presenta un consumo de alcohol a nivel promedio; así también se logró determinar que existen correlaciones entre el consumo de alcohol y las dimensiones de la variable estrés: síntomas fisiológicos $(\mathrm{r}=.358)$, síntomas de comportamiento social $(\mathrm{r}=.459)$, síntomas intelectuales y laborales $(\mathrm{r}=.482)$, y síntomas psicoemocionales ( $\mathrm{r}=.376)$. Por lo tanto, se concluye que existe correlación directa y significativa entre consumo de alcohol y estrés $(\mathrm{r}=0.500)$.
\end{abstract}

Palabras clave: Estrés, consumo de alcohol, trabajadora sexual, prostitución.

\begin{abstract}
The objective of this research was to determine the relationship between stress and alcohol consumption. The methodology corresponds to a non-experimental design of a correlational descriptive type; In which a population of 150 sex workers is involved in the referral center for sexually transmitted infections (CERITS) of the Carlos Monge Medrano Hospital in the city of Juliaca; However, it was decided to work with a sample of 103 participants chosen through non-probabilistic sampling. The instruments used were the stress questionnaire and questionnaire to identify disorders due to alcohol consumption AUDIT, both validated for the objectives of this research. The main results show that $43.7 \%$ present very high stress and $39.8 \%$ present average alcohol consumption; ( $\mathrm{R}=.358)$, symptoms of social behavior $(\mathrm{r}=.459)$, and intellectual and labor symptoms $(\mathrm{r}=.482)$. In addition, it was possible to determine that there are correlations between alcohol consumption and the dimensions of the stress variable: ), And psycho-emotional symptoms $(r=.376)$. Therefore, we conclude that there is a direct and significant correlation between alcohol consumption and stress $(r=0.500)$.
\end{abstract}

Keywords: Stress, alcohol consumption, sex worker, prostitution.

Correspondencia al autor:

email: oscar.benito@upeu.edu.pe 


\section{Introducción}

$\mathrm{Al}$ analizar el tema de prostitución es inevitable reconocer que este fenómeno actúa como un síntoma visible de la situación actual de la mujer dentro de una sociedad, especialmente en países donde prima una situación crítica a nivel político, económico y social, donde los pobres valores éticos y morales presentes van en aumento, donde es elevado el crecimiento de la pobreza, la falta de oportunidades laborales, y la creciente desigualdad social en la que viven (Urzua, 2005). Al respecto, en el Perú, desde el año 1911 el trabajo sexual es considerado como una actividad lícita para mujeres mayores de 18 años, siempre y cuando se realice bajo las condiciones que señalan las normas administrativas pertinentes; de lo contrario, se la considera una actividad ilícita, mas no sujeta a una sanción legal, a diferencia del proxenetismo que sí es considerado ilegal (Reaño, 2010). Precisamente, es de la anterior apreciación que podemos asimilar que las trabajadoras sexuales no tienen una identidad autónoma, pues su situación vital está subordinada a las reglas y normas sociales del contexto donde viven; de esta manera, las mujeres involucradas en el ejercicio sexual no están integradas socialmente, más bien viven inmersas en una cultura que valora las cosas materiales y devalúa el mundo de las personas; por ello, la pretendida libertad de la venta del cuerpo resulta desprotegida legalmente (Organización Mundial de la Salud, 2015).

Dejando de lado el aspecto legal y centrando nuestra atención del problema en términos de salud, es inevitable visualizar ampliamente las consecuencias que conlleva el ejercicio de la prostitución, pues tal como lo señalan Castellanos y Ranea (2014), en dicha actividad se han identificado principalmente efectos nocivos no solo para la salud física sino también para la salud mental, específicamente la prevalencia del estrés, siendo sus principales fuentes: el maltrato de los clientes, la preocupación por las enfermedades de transmisión sexual, el conocimiento de la familia acerca del trabajo que realizan, la sensación de llevar una doble vida y la vergüenza por la forma en que se ganan el dinero; por lo tanto, así como refiere Macías (2007) el estrés está relacionado con otras alteraciones de la salud que deterioran notablemente el funcionamiento normal, el bienestar y la calidad de vida de las personas que la padecen.

Aunado a todo lo dicho anteriormente, el problema se agrava cuando se descubre que una de las maneras de canalizar el estrés en el contexto de la prostitu- ción viene a ser el consumo de alcohol, obviamente no es el único; sin embargo, este factor toma fuerza pues estudios realizados como el de Rodríguez, Fuentes, Ramos, Gutiérrez, y Ruiz (2014) señalan que el 98\% de las trabajadoras sexuales consumen alcohol, precisando también que del mismo grupo, el 75\% de las trabajadoras sexuales se reportan como bebedoras a nivel muy alto, todo esto, como resultado de las fuentes de estrés. Sin embargo, lo crítico en este contexto se sitúa en las formas de consumo excesivo que implican un riesgo y un daño importante para el individuo, y en este punto nos referimos al consumo diario elevado, los episodios repetidos de beber hasta la intoxicación, el consumo asociado a factores de desestrés, consumo social y/o ocasional, pero en sí todos ocasionan un daño físico o mental, que posteriormente tiene como resultado el desarrollo de una dependencia o adicción (Flores, Huerta, Hernández, Páramo, y Morales, 2013).

Respecto al estrés. Este viene a ser un concepto que despierta gran interés no solo en la comunidad científica sino también en la población en general, donde es considerada como sinónimo de preocupación, de aquello que nos quita la tranquilidad y nos desestabiliza interiormente. Estos significados no difieren en demasía a los formulados por Hans Selye quien es considerado pionero en estudios del estrés, además fue él quien conceptualizó el término como un conjunto de reacciones fisiológicas del organismo a diversos agentes o factores nocivos del ambiente, tanto de naturaleza física o química (Montero, 2010). Al respecto, diversas investigaciones relacionadas con el estrés demuestran que las situaciones que afectan a las personas según el momento y su estado emocional son enfrentadas de distintas formas, pues cada persona posee un filtro que le permite excluir, aminorar o amplificar la percepción de la situación y sus demandas (Macías, 2007; Fonseca, 2008; Montero, 2010; Beltrán \& López, 2010); precisamente, este filtro está ligado a cada persona, de acuerdo a su carácter, personalidad, sensibilidad, modo de enfrentar los sucesos, experiencias pasadas, y sus mecanismos de defensa; lo cual, concuerda con los factores determinantes para enfrentar el estrés propuestos por Sandin, Chorot, Santed y Valiente (2002) quienes refieren que influyen en el individuo y su afrontamiento la forma de evaluar el suceso y/o las capacidades para hacerle frente, la manera de hacer frente a las dificultades, las características personales y la importancia de la percepción que la persona tiene del apoyo social. Otro punto importante a resaltar viene a ser la sociedad, como generadora de demandas estresantes 
para el individuo y la causante de coacciones a las formas en que se afrontan las circunstancias que se han clasificado como probables fuentes de estrés y como problemas potenciales de salud. Además, como constantes situaciones de estrés, se tiene la ejecución de múltiples tareas, sobrecarga de roles, conflictos interpersonales, perteneciendo a determinados grupos de estresores cotidianos, los cuales concuerdan con los principales estresores en las trabajadoras sexuales quienes refieren que el maltrato con los clientes, la preocupación por las enfermedades, el conocimiento de la familia acerca del trabajo que realizan, la sensación de llevar una doble vida, la relación con los hijos y la vergüenza por la forma en que se ganan el dinero, son sus principales fuentes estresoras e indirectamente uno de los motivos para justificar el consumo excesivo de alcohol (Moñino, 2012).

Respecto al consumo de alcohol. Esta es considerada la droga más consumida en el mundo; precisamente, América Latina se sitúa como la segunda región con mayor consumo per cápita de alcohol; y en este último contexto, Perú ocupa el sexto lugar. Respecto al tipo de alcohol consumido, nuestra cultura latina lo que más ingiere es cerveza (53\%); seguido de un $32,6 \%$ de licores como el vodka, whisky, y un $11,7 \%$ de vino (El Mundo, 2014). Es por ello que el consumo de alcohol es el principal factor de riesgo para la carga de morbilidad en países en vías de desarrollo de las Américas, y el segundo factor de riesgo en los países en vías de desarrollo de la región. A pesar de que se observan diferencias regionales, el consumo de alcohol en el continente americano fue en promedio más de 50\% mayor que en el mundo en general; asimismo, se advierte la tendencia a beber en exceso de manera descontrolada (Rehm \& Monteiro, 2005). Para la Organización Panamericana de la Salud (2008), la presencia de la cultura del alcohol fue consolidándose durante siglos en todas las capas sociales; se enseña desde la infancia, no solo cómo usar el alcohol, sino también, dentro de ciertos límites, cómo no abusar de él. Así, el hecho de que tomar alcohol sea normal, contribuye también a enmascarar y a normalizar sus efectos y lleva a no exhibir públicamente sus consecuencias; puesto que, el sujeto que bebe moderadamente se siente aceptado en su ambiente si no exagera, y el ambiente en general es muy tolerante, y ello le estimula a no perder tal aceptación con comportamientos cuando menos molestos. Sin embargo, el problema surge cuando se descubre que un número importante de usuarios desarrolla dependencia a esta sustancia; es por ello, que la prevalencia se presenta actualmente en línea ascendente a nivel mundial; precisamente, al hablar de dependencia no podemos dejar de lado el término adicción, el cual engloba dos conceptos: la dependencia física y psicológica. Sobre esto último, se enfatiza respecto al ámbito psicológico las actividades de búsqueda de la sustancia y la evidencia de patrones de uso patológico, y en la parte física resalta los múltiples episodios de uso de la sustancia (Salazar, Ugarte, Vásquez, \& Loaisa, 2004).

En nuestro contexto, el problema de consumo del alcohol en el Perú, está tomando dimensiones cada vez más complejas y está complicando la vida de miles de ciudadanos, tal como lo reportan el Centro de Información y Educación para la Prevención del Abuso de Drogas (CEDRO, 2006) y la Comisión Nacional para el Desarrollo y Vida sin Drogas (DEVIDA, 2003), entidades que refieren que para la reducción de la demanda implica trabajar en el ámbito preventivo, buscando modificar las condiciones que promueven el incremento del número de consumidores, y en base a dicha información es posible establecer qué poblaciones están en mayor riesgo de involucrarse en el consumo descontrolado, las mismas que se convierten en grupo objetivo de las acciones preventivas. Principalmente las personas que residen en sectores urbano marginales o las familias que tienen miembros consumidores son un ejemplo de poblaciones que reciben atención en la mayor parte de programas preventivos en el país; y de este grupo, no se puede dejar de lado a las trabajadoras sexuales. Por lo tanto, no podemos negar que el trabajo sexual es un tema que se encuentra lleno de mitos y estigmas sociales, pues es más complejo de lo que se podría pensar; sin embargo, una mirada más profunda y completa sobre este hecho nos permite comprender el mundo subjetivo de las personas involucradas, puesto que las situaciones estresantes que conllevan demandas conductuales pueden originar problemas psicológicos.

\section{Método}

Esta investigación se encuadra en un diseño no experimental, de tipo descriptivo correlacional y de corte transversal transeccional, pues se buscó relacionar las variables sin el afán de manipular algunas de ellas, además se recogió los datos en un solo momento en el tiempo (Hernández, Fernández, \& Baptista, 2010).

\section{Participantes}

La población estuvo constituida por 150 trabajadoras sexuales que acuden al centro de CERITS del Hospital Carlos Monge Medrano de la ciudad de Juliaca; sin embargo, por razones de accesibilidad se trabajó con 
una muestra de 103 trabajadoras sexuales resultantes de un muestreo no probabilístico, que respondieron a los cuestionarios después de haber sido informadas y consentidas en participar.

\section{Instrumentos}

Para la variable estrés se utilizó el cuestionario de estrés tercera versión, el cual consta de 31 ítems distribuidos en cuatro dimensiones (síntomas fisiológicos, de comportamiento social, intelectual y laboral, y psicoemocionales), el mismo que es un instrumento de naturaleza cuantitativa con una escala de medida discreta con amplitud colectiva; así también, este instrumento informa y clasifica los niveles de estrés en: muy alto, alto, medio, bajo y muy bajo. En cambio, para la variable consumo de alcohol se utilizó el cuestionario de identificación de los trastornos debidos al consumo de alcohol (AUDIT) que consta de 10 ítems, el cual es un instrumento de evaluación desarrollado y propuesto por la Organización Mundial de la Salud como un método simple para evaluar el consumo de alcohol en el ámbito de la atención primaria a nivel internacional, pues ayuda a identificar si la persona presenta un consumo de riesgo, un consumo perjudicial o una dependencia de alcohol.

Respecto a la validez y confiabilidad de los instrumentos, ambos fueron contextualizados para la zona y se ha realizado el cálculo de la confiabilidad mediante el coeficiente Alfa de Cronbach hallando para el cuestionario de estrés un valor de 0.924 y para el cuestionario AUDIT un valor de 0.851; siendo estos valores indicadores de una elevada confiabilidad. La muestra para los cálculos de confiabilidad fueron 33 trabajadores de la empresa SHEMICO I.E.R.L. de la ciudad de Juliaca.

\section{Análisis de datos}

Para el análisis de datos se utilizó el paquete estadístico SPSS versión 22.0; asimismo, se trabajó con estadísticos descriptivos como son las tablas de frecuencias y estadísticos de correlación, como es el coeficiente de correlación de Pearson.

\section{Resultados y Discusión}

\section{Análisis descriptivo de la variable estrés:}

En la tabla 1 se puede apreciar que el $43.7 \%$ de trabajadoras sexuales presentan un nivel de estrés muy alto, es decir, que la cantidad de síntomas y su frecuencia de presentación es indicativa de una respuesta de estrés severa y perjudicial para la salud y que requieren intervención inmediata; así también, el $25.2 \%$ presenta un nivel de estrés medio, seguido del $13.6 \%$ con niveles de estrés bajo y alto respectivamente y finalmente solo el $3.9 \%$ de las trabajadoras sexuales presenta un nivel de estrés muy bajo, indicando la ausencia de síntomas de estrés u ocurrencia muy rara que no amerita desarrollar actividades de intervención.

Tabla 1

Nivel de estrés que presentan las trabajadoras sexuales atendidas en el Centro de Referencia de Infecciones de Transmisión Sexual (CERITS) del Hospital Carlos Monge Medrano de la ciudad de Juliaca.

\begin{tabular}{lcc}
\hline \multicolumn{1}{r}{ Nivel } & Frecuencia & \% \\
\hline Muy bajo & 4 & 3.9 \\
Bajo & 14 & 13.6 \\
Medio & 26 & 25.2 \\
Alto & 14 & 13.6 \\
Muy alto & 45 & 43.7 \\
Total & 103 & 100.0 \\
\hline
\end{tabular}

Análisis descriptivo de la variable consumo de alcohol:

La tabla 2 muestra el nivel de consumo de alcohol que presentan las trabajadoras sexuales, y se evidencia que el 39.8\% de ellas presenta un nivel promedio de consumo, seguido del 31.1\% con un nivel de consumo bajo y, finalmente, el $29.1 \%$ presenta un nivel de consumo alto.

Tabla 2

Nivel de consumo de alcohol de las trabajadoras sexuales atendidas en el Centro de Referencia de Infecciones de Transmisión Sexual (CERITS) del Hospital Carlos Monge Medrano de la ciudad de Juliaca.

\begin{tabular}{ccc}
\hline Nivel & Frecuencia & \% \\
\hline Bajo & 32 & 31.1 \\
Medio & 41 & 39.8 \\
Alto & 30 & 29.1 \\
Total & 103 & 100.0 \\
\hline
\end{tabular}

\section{Análisis de correlación de las variables estrés y con- sumo de alcohol}

Según la tabla 3, se puede apreciar el análisis de correlación, el cual determina la existencia de una relación directa y significativa entre el estrés y consumo de alcohol $(\mathrm{r}=.500)$, siendo el coeficiente de determinación (r2 = .250) que indica que la variación del consumo de alcohol se explica por la variación del nivel de estrés en un $25.0 \%$, lo cual es muy significativo. 
Mamani, O. Coaquira, J. y Sapillado, M.

Tabla 3

Resumen del modelo de correlación y determinación de Pearson entre estrés y consumo de alcohol en trabajadoras sexuales.

\begin{tabular}{ccccc}
\hline Modelo & $\mathbf{R}$ & R cuadrado & R cuadrado corregida & Error típ. de la estimación \\
\hline 1 & $.500($ a) & .250 & .243 & 6.10688 \\
\hline Nota: a Variables predictoras: Constante, Estrés & &
\end{tabular}

Análisis de correlación de las variables según dimensiones:

Seguidamente, en la tabla 4, podemos apreciar el análisis de correlación entre la variable consumo de alcohol y las cuatro dimensiones de la variable estrés: síntomas fisiológicos $(\mathrm{r}=.358 ; \mathrm{p}<0.05)$, síntomas de comportamiento social $(r=.459 ; \mathrm{p}<0.05)$, los síntomas intelectuales y laborales $(\mathrm{r}=.482 ; \mathrm{p}<0.05)$, y síntomas psicoemocionales $(\mathrm{r}=.376 \mathrm{p}<0.05)$, las cuales son directas y significativas.

Tabla 4

Análisis de correlación entre la variable consumo de alcohol y las dimensiones de la variable estrés.

\begin{tabular}{cccccc}
\hline Variable & & $\begin{array}{c}\text { Síntomas } \\
\text { fisiológicos }\end{array}$ & $\begin{array}{c}\text { Síntomas de } \\
\text { comportamiento } \\
\text { social }\end{array}$ & $\begin{array}{c}\text { Síntomas } \\
\text { intelectuales y } \\
\text { laborales }\end{array}$ & $\begin{array}{c}\text { Síntomas } \\
\text { psicoemocionales }\end{array}$ \\
\hline $\begin{array}{c}\text { Consumo de } \\
\text { Alcohol }\end{array}$ & $\begin{array}{c}\text { Correlación de } \\
\text { Pearson }\end{array}$ & $.358\left(^{* *}\right)$ & $.459\left(^{* *}\right)$ & $.482(* *)$ & $.376(* *)$ \\
& Sig. (bilateral) & .000 & .000 & .000 & .000 \\
& $\mathrm{~N}$ & 103 & 103 & 103 & 103 \\
\hline
\end{tabular}

\section{Discusión}

En el presente estudio se halló la presencia de niveles elevados de estrés experimentados por las trabajadoras sexuales (43.7\%). Este resultado concuerda con los hallazgos de Reaño (2010), Beltrán \& López (2010), y Salinas, Padilla, Ruiz, \& Vera (2014) quienes encontraron también una prevalencia del estrés en mujeres dedicadas al trabajo sexual. Así también, se halló en este estudio un nivel promedio de consumo de alcohol por parte de las trabajadoras sexuales (39.8\%), siendo estos resultados similares a los encontrados por Rodríguez et al., (2014), y Salinas et al., (2014), quienes hallaron que el 75\% de mujeres que ejercen la prostitución se reportan como bebedoras en un nivel alto.

Se podría decir entonces que uno de los primeros aportes de esta investigación viene a ser el hecho de corroborar la literatura científica respecto a la prevalencia de niveles elevados de estrés y consumo excesivo de alcohol en mujeres que ejercen el trabajo sexual.

Así también, los resultados aún más importantes de esta investigación resaltan al exponer los hallazgos de correlaciones significativas entre el consumo de alcohol y las dimensiones de la variable estrés: síntomas intelectuales y laborales ( $\mathrm{r}=0.482$ ), síntomas de com- portamiento social ( $(\mathrm{r}=0.459)$, síntomas psicoemocionales $(\mathrm{r}=0.376)$, síntomas fisiológicos $(\mathrm{r}=0.358)$, lo cual nos da a entender que si existe una correlación directa y significativa entre los niveles de consumo de alcohol y las manifestaciones de estrés. Al respecto, debido a las escasas referencias en la literatura respecto a estudios que relacionan ambas variables, es que este estudio viene a ser una clara manifestación científica de la relación entre el consumo del alcohol y el estrés. Asimismo, los hallazgos obtenidos pueden ser explicados si se toman en cuenta que las fuentes de estrés, tales como el maltrato del cliente, las características de las personas que solicitan el servicio, los pedidos y exigencias de riesgo durante el intercambio sexual, hacen vulnerables a las trabajadoras sexuales, recurriendo estas, al consumo de alcohol como única estrategia de desestrés, o en el peor de los casos como refugio ante su situación (Reaño, 2010). Desde la perspectiva de la iniciación en el consumo de alcohol, y en concordancia con los hallazgos de Rodríguez et al., (2014) quienes refieren que el 97\% de trabajadoras sexuales informa que son los clientes quienes las obligan a beber alcohol. Otro grupo similar refiere que son los encargados de los lugares de trabajo quienes obligan el consumo forzoso, es lo que nos lleva a interpretar que existe un ambiente de violencia; y son precisamente estas condiciones, las cuales son frecuentes en su ambiente de trabajo y que es ejercido por múltiples actores, lo que nos lleva a 
asimilar que el consumo de alcohol y drogas se asocia con esta violencia, por lo que debe explorarse más este vínculo en futuros episodios.

\section{Conclusiones}

Tal como se ha podido apreciar y deliberar en párrafos anteriores este estudio halló la prevalencia de niveles elevados de estrés (43.7\%) y consumo de alcohol por parte de trabajadoras sexuales que acuden al Centro de Referencia de Infecciones de Transmisión Sexual (CERITS) del Hospital Carlos Monge Medrano de la ciudad de Juliaca. Asimismo, se ha encontrado una correlación directa y significativa entre la variable consumo de alcohol y estrés $(\mathrm{r}=0.500)$; así como correlaciones entre el consumo de alcohol y las dimensiones de estrés: síntomas intelectuales y laborales $(r=0.482)$, síntomas de comportamiento social $((\mathrm{r}=0.459)$, síntomas psicoemocionales $(\mathrm{r}=0.376)$, síntomas fisiológicos $(\mathrm{r}=0.358)$.

\section{Referencias}

Beltrán, A. J., \& López, J. D. (2010). Comprensión desde las historias de la vida de los estilos de afrontamiento ante el estrés en un grupo de trabajadoras sexuales de Bogotá. Psychologia: Avances de la disciplina, 4(2), 131-141.

Castellanos, E., \& Ranea, B. (2014). La perspectiva de género y de los derechos humanos en el análisis de la prostitución y la trata de mujeres con fines de explotación sexual: con una aproximación de la voz propia de las mujeres. Dilemata, 6(16), 161-179.

CEDRO- Centro de Información y Educación para la Prevención del Abuso de Drogas. (2006). El problema de las drogas en el Perú. Lima- Perú: CEDRO.

DEVIDA Comisión Nacional para el Desarrollo y Vida sin Drogas. (2003). Encuesta Nacional de Prevención y Consumo de Drogas 2002. Perú: proyecto RLA/AD/ PER/99/D77.

El Mundo. (12 de mayo de 2014). Economía \& Negocios El Mundo. Venezuela es el tercer país con mayor consumo de alcohol en la región. Obtenido de http://www. elmundo.com.ve/noticias/negocios/consumo/venezuela-es-el-tercer-pais-con-mayor-consumo-de-a.aspx

Flores, C., Huerta, M. R., Hernández, J., Páramo, D., \& Morales, I. (2013). Prevalencia de Alcoholismo en Trabajadores de la Industria del Cuero-Calzado y su Asociación con el Nivel de Desestrés. Ciencia \& Tra- bajo, 15(47). Recuperado el 28 de agosto de 2015, de http://www.scielo.cl/scielo.php?script=sci_arttext\&pi$\mathrm{d}=$ S0718-24492013000200006

Fonseca, Y. (2008). El estrés psicológico y sus principales formas de expresión en el ámbito laboral. Informe de investigación, Universidad de La Habana, Facultad de Psicología, La Habana.

Hernández Sampieri, R., Fernández Collado, C., \& Baptista Lucio, M. (2010). Metodología de la investigación. México D.F.: McGraw Hill.

Macías, H. (2007). Estudio de los niveles de estrés del contingente español desplegado en Irak en misión de mantenimiento de paz. Tesis para optar el grado de Doctor en Psicología, Universidad de Granada, Facultad de Psicología.

Montero, G. (2010). Nivel del estrés laboral y patrón de conducta en los empleados de la Universidad Nacional de Educación Enrique Guzmán y Valle. Informe de investigación. Vicerrectorado Académico, Universidad Nacional de Educación Enrique Guzmán y Valle La Cantuta, Dirección del Instituto de Investigación, Lima, Perú.

Moñino, M. (2012). Factores sociales relacionados con el consumo de alcohol en adolescentes de la Región de Murcia. Memoria presentada para optar el grado de Doctor, Universidad de Murcia, Facultad de Medicina, Murcia, España.

OMS. (2015). Implementación de programas integrales de VIH/ITS con personas trabajadoras del sexo: enfoques prácticos basados en intervenciones colaborativas. OMS. Recuperado el 22 de septiembre de 2015, de http://apps.who.int/iris/ bitstream/10665/176025/1/9789243506180_spa.pdf?ua $=1 \& u a=1$

OPS. (2008). Alcohol y atención primaria de la salud. Washington D.C.: OPS.

Reaño, M. P. (2010). Fuentes de estrés en un grupo de trabajadoras sexuales. Tesis para optar por el título de Licenciada en Psicología con mención en Psicología Clínica, Pontificia Universidad Católica del Perú, Facultad de Letras y Humanidades.

Rehm, J., \& Monteiro, M. (2005). El consumo de alcohol y la carga de morbilidad en el continente americano: implicaciones para las políticas de control del consumo de alcohol. Revista Panamericana de Salud Pública, 18(4-5), 241-248. Recuperado en abril de 2015, de http://www.scielosp.org/scielo.php?script=sci_arttext\&pid=S1020-49892005000900003

Rodríguez, E., Fuentes, P., Ramos-Lira, L., Gutiérrez, R., \& Ruiz, E. (2014). Violencia en el entorno laboral del trabajo sexual y consumo de sustancias en mujeres mexicanas. Salud Mental, 37(4), 355-360. 
Mamani, O. Coaquira, J. y Sapillado, M.

Salazar, E., Ugarte, M., Vásquez , L., \& Loaisa, J. (2004). Consumo de Alcohol y Drogas y Factores Psicosociales Asociados en Adolescentes. Anales de la Facultad de Medicina , 179-188.

Salinas, C. E., Padilla, N., Ruiz, M. L., \& Vera, A. M. (2014). La autoestima como factor estresor intrapersonal para el consumo de alcohol en trabajadoras sexuales. Enfermería Global, 13(33). Recuperado el 12 de agosto de 2015, de http://scielo.isciii.es/scielo. php?pid=S1695-61412014000100007\&script=sci_arttext\&tlng=en
Sandin, B., Chorot, P., Santed, M. A., \& Valiente, R. M. (2002). Estrés y salud: relación de los sucesos vitales y el estrés diario con la sintomatología somática y la enfermedad. Revista electrónica Ansiedad y Estrés, 8(1), 73-87.

Urzua, K. (agosto de 2005). Diálogos, propuestas, historias para una Ciudadanía Mundial. Recuperado el 25 de agosto de 2015, de La otra cara de la prostitución: http://base.d-p-h.info/es/fiches/dph/fiche-dph-6666. html 